\title{
On the Energy Efficiency of Survivable Optical Transport Networks with Flexible-grid
}

\author{
Jorge López ${ }^{(1,3)}$, Yabin Ye ${ }^{(1)}$, Víctor López ${ }^{(2)}$, Felipe Jiménez ${ }^{(2)}$, Raúl Duque ${ }^{(2)}$, Peter M. Krummrich ${ }^{(3)}$ \\ (1) Huawei Technologies Duesseldorf GmbH, European Research Center, Riesstr. 25, 80992 Munich, \\ Germany, E-mail address: jorge.vizcaino@huawei.com \\ (2) Telefónica I+D, c/ Don Ramón de la Cruz, 84, 28006 Madrid, Spain \\ (3) Technisque Universitaet Dortmund, Friedrich-Woehler-Weg 4, 44227 Dortmund, Germany
}

Abstract An energy efficiency comparison of conventional path protection schemes for fixed-grid WDM and flexible-grid OFDM-based networks has been carried out. The survivable elastic network with SP scheme was found to offer the best energy efficiency per $\mathrm{GHz}$ at any traffic load value.

\section{Introduction}

Telecommunications operators are continuously exploring new alternatives to upgrade their networks in order to handle the ever increasing Internet traffic demand. However, a capacity upgrade is often accompanied by an increase in energy consumption, which affects not only the operational expenditures (OPEX) of the telecom carriers, but also their carbon footprint. In this scenario, energy efficiency is becoming one of the key design parameters for the planning and the operation of future networks, in which the optical transport network will play an essential role to handle the new capacity requirements. These networks, based on Dense Wavelength Division Multiplexing (DWDM) technologies, traditionally operate with a single line rate (SLR), dictated by the available transponders at the time of installation, and a channel spacing specified by the ITU-T grid (usually $50 \mathrm{GHz}$ ). Considering a single-layer network design, this rigid resource allocation may lead to some inefficient use of the spectral resources and also to some energy wastage, as the actual traffic demand may be much lower than the allocated wavelength capacity. Several alternatives have been proposed to overcome this problem and enhance the resource allocation flexibility. Thus, the introduction of mixed line rate (MLR) operation can be seen as a middle-term solution to support heterogeneous traffic requests with different rate requirements. Then, for the longterm, the flexible-grid or elastic-bandwidth optical network appears as an interesting solution since it allows the expansion or contraction of the channel bandwidth according to the demand. For this elastic network, COOFDM (Coherent Optical- Orthogonal Frequency Division Multiplexing) is considered as a promising technology for transmission.

Nowadays, telecommunication networks are indispensable for the availability of many services in our society, and hence it is necessary to guarantee a high resilience, which can be provided by protection. Protection is becoming even more critical as the traffic carried by a single fiber increases (i.e. a single fiber cut might interrupt a large amount of traffic). Many protection schemes have been proposed so far, but in most of the cases the energy efficiency has not been taken into account in the performance evaluation. In our previous publication ${ }^{1}$, we showed the benefits in energy efficiency of an Elastic OFDM-based network compared to the conventional WDM networks (SLR and MLR). The present paper aims at complementing our previous analysis, by evaluating the energy efficiency of three common path protection schemes: $1+1$ dedicated protection $(1+1 D P), 1: 1$ dedicated protection $(1: 1 D P)$, and shared protection $(S P)$.

\section{Network considerations}

WDM network: A $50 \mathrm{GHz}$ grid with 80 wavelength in the $\mathrm{C}$-band and line rates of 10 $\mathrm{Gb} / \mathrm{s}$ (NRZ-OOK), $40 \mathrm{~Gb} / \mathrm{s}$ (DQPSK), and 100 $\mathrm{Gb} / \mathrm{s}$ (PDM-QPSK), with reaches ${ }^{2}$ of 3200 , 2200 , and $1880 \mathrm{~km}$ respectively, have been adopted. Two types of operation are considered: SLR (10, 40 or $100 \mathrm{~Gb} / \mathrm{s})$ and MLR (10/40/100 $\mathrm{Gb} / \mathrm{s})$. In this latter approach, in order to minimize the cross-talk effect between adjacent channels of different technologies, the C-band is divided into two wavebands, separated by a guard band of $200 \mathrm{GHz}$ : a first one for $10 \mathrm{~Gb} / \mathrm{s}$, and a second one for both 40 and $100 \mathrm{~Gb} / \mathrm{s}$. Elastic network: A frequency slot of $12.5 \mathrm{GHz}$ has been considered, so the transmission rate of a single subcarrier can be 12.5, 25, 37.5, 50, 62.5 and $75 \mathrm{~Gb} / \mathrm{s}$ for BPSK, QPSK, 8QAM, 16QAM, 32QAM, and 64QAM respectively. Several subcarriers can be combined to create super-channels with higher transmission rate. A guard band of two subcarriers $(25 \mathrm{GHz})$ is used to separate adjacent channels. A transmission reach $^{1}$ of $4000,2000,1000,500,250$ and 125 $\mathrm{km}$ has been assumed for BPSK, QPSK, 8QAM, 16QAM, 32QAM, and 64QAM respectively. 
Power consumption of network elements a) Transponders: 34,98 and $351 \mathrm{~W}^{3}$ have been assumed for WDM transponders of 10, 40 and $100 \mathrm{~Gb} / \mathrm{s}$ transponders respectively. Due to the commercial unavailability of CO-OFDM transponders, some assumptions have been made to estimate realistic values of power consumption ${ }^{1}$. The presence of DSP (Digital Signal Processing) at the transmitter part is assumed to be the main distinction between a CO-OFDM transponder and a coherent WDM one, and therefore the comparison could be based on the DSP complexity. Since this complexity is similar at the same bit rate ${ }^{4}$, the power consumption has been assumed to be the same for both types of transponders. Accordingly, based on the values of the dual polarization coherent transponders ${ }^{3}$ of 250 and $351 \mathrm{~W}$ for 40 and $100 \mathrm{~Gb} / \mathrm{s}$ respectively (125 and $175.5 \mathrm{~W}$ for single polarization); and assuming that the DSP scales linearly with the bit rate, the power consumption of a single polarization CO-OFDM transponder can be interpolated as a function of its transmission rate (1). Tab. 1 presents the power consumption (PC) values for the different modulation formats. An additional $20 \%$ of $\mathrm{PC}$ is considered as the overhead contribution for each transponder type. $P C_{\text {OFDM }}(W)=1.683 \cdot T R(G b / s)+91.333$

b) Optical Cross Connects (OXC): A flexible-grid OXC was assumed to consume similar power as the fixed-grid variant: dependent on the node degree $N$ and the add/drop degree $\alpha$ as in (2) ${ }^{3}$.

$$
P C_{\text {OXC }}(\mathrm{W})=N \cdot 85+\alpha \cdot 100+150
$$

c) Optical Amplifiers (OA): An EDFA (Erbium Doped Fiber Amplifier) consuming $30 \mathrm{~W}^{3}$ per direction, and an overhead contribution of 140 W per amplifier location, has been considered.

\section{Survivable-resource allocation algorithms}

We studied the resource allocation for a set of static demands that is resilient to any single link failure, the dominating form of failure in optical networks. The heuristic methodology used for the resource allocation in a static scenario ${ }^{2}$ has been complemented by considering different protection schemes (pre-planned backup routes are calculated offline). In these algorithms, the demands from the traffic matrix are firstly sorted

Tab. 1: Power Consumption of a CO-OFDM transponder for different modulation formats

\begin{tabular}{|c|c|c|}
\hline Mod. Format & Subc. Cap.(Gb/s) & P. Cons. (W) \\
\hline BPSK & 12.5 & 112.374 \\
\hline QPSK & 25 & 133.416 \\
\hline 8QAM & 37.5 & 154.457 \\
\hline 16QAM & 50 & 175.498 \\
\hline 32QAM & 62.5 & 196.539 \\
\hline 64QAM & 75 & 217.581 \\
\hline
\end{tabular}

in descending order with the highest demand first (similar results were obtained by ordering with the product of the traffic demand value and the shortest path length). Then, the resource allocation for each demand from the list is evaluated according to the protection scheme:

a) Dedicated path protection (1+1 and 1:1): the allocation is jointly evaluated for the possible combinations of candidate working path ( $k$ shortest paths), and their corresponding candidate backup paths (its $k$ link-disjoint paths). For the feasible path-pair combinations, a metric based on the power consumption is calculated, allowing for the selection of the most energy efficient path-pair for the allocation of resources. If a demand cannot be served with a working and backup path, then it is blocked. In both the $1+1$ and 1:1 schemes, the spectral resources for the working and backup paths are reserved and pre-cross-connected. The difference lies in the computation of the total power consumption, as in the $1+1$ scheme the transmission is simultaneous in both working and backup paths, whereas in the 1:1 scheme the transmission occurs only in the working path.

b) Shared path protection: Once the resource allocation is evaluated for all the traffic demands in their working paths, the remaining spectral resources can be shared by any backup path. Since the goal is to provide protection against any single link failure (i.e. assuming that only one link will fail at the same time), the failure of each link in the network has been analyzed consecutively. For each link failure, the traffic demands are listed, arranged in descending order of their traffic demand value, and then the resource allocation is checked again for the listed demands from source to destination without considering the failed link (i.e. the link is pruned from the network graph). If a backup route can be provided for a demand, it is stored in the list of backup routes, otherwise it is considered as blocked because it cannot be protected against failure of this link. This scheme is failure-dependent, since multiple backup paths are associated with a working path and the selection of one or another depends on which particular link has failed. On the other hand, the dedicated protection schemes are failure-independent as the same backup is used independently of the link failure.

Besides the energy efficiency measure (traffic transported/power consumption), the spectral efficiency is also a relevant parameter to consider. Therefore, we adopt a measure from wireless ${ }^{5}$, Energy efficiency per $\mathrm{GHz}$ (bits/Joule/GHz) to account for both parameters: 


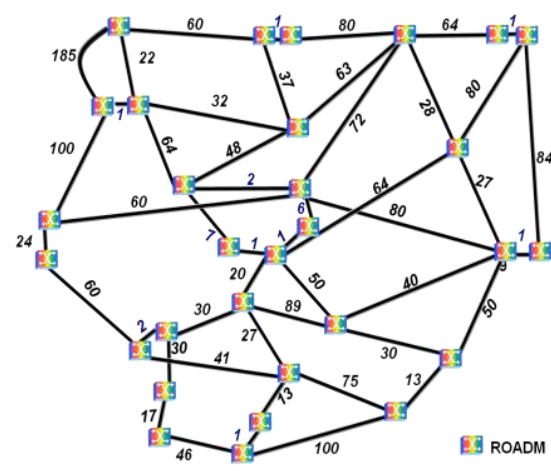

Fig. 1: Spanish core network of Telefónica. EnergyEfficiency(bits/Joule)

AvgSpectrumOccupancy* BandwidthCBand $(\mathrm{GHz})$

\section{Simulation Results}

The studied network topology is the Spanish core network of Telefónica shown in Fig.1 (30 nodes, 96 bidirectional links), with a realistic traffic matrix for 2012 scaled up to a factor of 20 to obtain a total traffic ranging from 3.22 to $64.48 \mathrm{~Tb} / \mathrm{s}$. A single fiber pair per link and transparent connectivity have been considered.

Among the different network types of operation, the SLR 40G is generally the most energy efficient at low traffic load, due to its lower energy per bit at the transponder $(2.45$ bits/Joule compared to $3.4,3.51$ and 9 for $10 \mathrm{G}$, $100 \mathrm{G}$ and BPSK in the OFDM, respectively). However, when the traffic increases, the spectrum occupancy in the links becomes considerably high, resulting in some blocked demands, either due to the unavailability of spectral resources or to the impossibility of transparent communication. As the traffic grows, the energy efficiency of the SLR 100G, MLR, and especially of the Elastic network is improved. Moreover, at high traffic load, the spectral efficiency also starts to become relevant, since it affects the maximum capacity that the network is able to handle with a single fiber pair (Tab. 2). This fact is also important from the energy efficiency point of view, since deploying additional network elements not only implies an increase in cost, but also in power consumption. The Elastic network clearly shows the lowest spectrum occupancy and blocking ratio thanks to its distance adaptive modulation and its flexible grid operation. Regarding the different protection schemes, $S P$ and 1:1 DP

Tab. 2: Max. Traffic Supported with no blocking

\begin{tabular}{|c|c|c|}
\hline Network Type & $\begin{array}{c}\text { Max. Traffic } \\
\text { with DP (Tb/s) }\end{array}$ & $\begin{array}{c}\text { Max. Traffic } \\
\text { with SP (Tb/s) }\end{array}$ \\
\hline Elastic & 54.808 & 61.256 \\
\hline SLR 10 Gb/s & 3.224 & 3.224 \\
\hline SLR $40 \mathrm{~Gb} / \mathrm{s}$ & 12.896 & 16.12 \\
\hline SLR $100 \mathrm{~Gb} / \mathrm{s}$ & 32.24 & 41.912 \\
\hline MLR & 32.24 & 45.136 \\
\hline
\end{tabular}

Fig. 2: Energy efficiency per $\mathrm{GHz}$.

show better energy efficiency than $1+1 D P$, as the backup paths only consume energy in case of failure. However, the SP scheme offers lower spectrum occupancy and blocking ratio than the 1:1 DP one as the spectral resources are shared by different backup paths.

Fig.2 shows the results concerning Energy Efficiency per $\mathrm{GHz}$ for the different types of network operation and protection schemes, at different traffic load conditions with no blocking conditions (all the traffic demands are protected against any single link failure). Therefore, it presents an overview of the energy efficiency, spectrum occupancy and blocking ratio measures. As shown, a survivable Elastic network with $S P$ scheme offers the best results in energy efficiency per $\mathrm{GHz}$ at any traffic load value. Simulations were carried out in another country-sized network, the Deutsche Telekom network, leading to similar results.

\section{Conclusions}

The Elastic OFDM-based network is proposed as a promising candidate for the operation of future optical transport networks, and survivability is certainly a parameter that needs to be considered. The resource allocation flexibility of the Elastic network can be beneficial in energy efficiency for a realistic network scenario with different protection schemes. In particular, a shared protection scheme can offer significantly better results than the dedicated ones $(1+1,1: 1)$. In conclusion, this paper presents more reasons to adopt the Elastic network approach for the Network of the Future.

This work was partially supported by the EU FP7 funded projects TREND and CHRON.

\section{References}

[1] J.López Vizcaíno et al., Computer Networks, doi: 10.1016/j.comnet.2012.03.012. (2012)

[2] A.Klekamp et al.,Proc.OFC'11, NMC5 (2011)

[3] F.Smyth et al., "GreenTouch Draft Report on Baseline Power Consumption," v.1.8, (2011)

[4] S.J.Savory, Proc. OFC'08, OTuO3 (2008)

[5] R.Xie et al., Proc.INFOCOM'12, TS45 (2012) 